Check for updates

Cite this: RSC Adv., 2017, 7, 31408

\title{
Intestinal absorption and neuroprotective effects of kaempferol-3-O-rutinoside
}

\author{
Yingcong Ma, (D) a Yujie Liu, (D) a Aning Sun, (D) ${ }^{a}$ Yitian Du, (D) ${ }^{a}$ Min Ye, (D) b \\ Xiaoping Pu (iD c and Xianrong Qi (iD *a
}

Kaempferol-3-O-rutinoside (K3R) has been proven to have biological activities for the prevention and treatment of central nervous system (CNS) diseases. However, the details of its absorption from the gastrointestinal tract and transport across the blood-brain barrier (BBB) are not clear. Therefore, to provide a basis for its efficacy, we explored its intestinal absorption with Caco-2 monolayer cells, rat orthotopic intestinal perfusion, its transport across a BBB model based on bEnd.3 monolayer cells and its pharmacological effects on PC12 cells in terms of neuropathy. The results indicated that the transport of K3R is concentration dependent; moreover, the apparent permeability coefficient $\left(P_{\text {app }}\right)$ of K3R lacked directionality, and the efflux rate after adding $\mathrm{P}$-glycoprotein inhibitor did not show a significant change. K3R was found to be completely absorbed in the intestines of rats, and the absorption process follows the first-order kinetics. K3R can pass through the BBB with passive diffusion. The neuroprotective effects of K3R were related to stabilization of the mitochondrial membrane and a decrease in reactive oxygen species. These results demonstrated that K3R can be absorbed in a relatively moderate ratio in the gastrointestinal tract and transported to the brain. Thus, K3R may be a potent drug for the prevention and treatment of neurodegenerative diseases such as Parkinson's disease.

Received 13th May 2017
Accepted 1st June 2017
DOI: 10.1039/c7ra05415g
rsc.li/rsc-advances

\section{Introduction}

Kaempferol-3-O-rutinoside (K3R) is an effective component that is present in some medicinal plants, such as Carthamus tinctorius. It is a flavonoid glycoside and takes the form of a yellow amorphous powder. K3R has been reported to have a variety of biological activities, such as anti-oxidation, protection of the liver and gallbladder, antibacterial, anti-inflammatory, inhibition of uric acid, anti-glycation, prevention of kidney stones and promotion of wound healing. ${ }^{1-8}$ In recent years, the efficacy of $\mathrm{K} 3 \mathrm{R}$ in the central nervous system (CNS) has been studied. It has been reported that $\mathrm{K} 3 \mathrm{R}$ can prevent and treat ischemic brain damage. ${ }^{9}$ It can also treat neuronal inflammation by inhibiting the activation of transcription factor nuclear factor$\kappa \mathrm{B}(\mathrm{NF}-\kappa \mathrm{B})$, a signal transducer and activator of transcription 3 (STAT3). ${ }^{10}$ Further studies have shown that K3R can significantly reduce the neurotoxicity of beta-amyloid-induced nerve cells. ${ }^{11}$ For multi-infarct dementia model rats, K3R can improve rat memory function and reduce damage due to oxidative stress

${ }^{a}$ Beijing Key Laboratory of Molecular Pharmaceutics and New Drug Delivery System, Department of Pharmaceutics, School of Pharmaceutical Sciences, Peking University, Beijing, 100191, PR China. E-mail: qixr@bjmu.edu.cn; Fax: +86 10 82801584; Tel: +861082801584

${ }^{b}$ Department of Natural Medicines, School of Pharmaceutical Sciences, Peking University, Beijing, 100191, PR China

${ }^{c}$ Department of Molecular and Cellular Pharmacology, School of Pharmaceutical Sciences, Peking University, Beijing, 100191, PR China on the nervous system. ${ }^{12}$ Our previous studies have shown that safflower flavonoid extract (in which $\mathrm{K} 3 \mathrm{R}$ is the main ingredient) is effective in preventing and treating Parkinson's disease (PD), and the protective mechanism of K3R on nerve cells corresponds to specific binding to $\mathrm{DJ}-1 .^{\mathbf{1 3 , 1 4}}$ However, the absorption of K3R from the gastrointestinal tract into the blood and then across the blood-brain barrier (BBB) is poorly understood. Therefore, it is important to study the absorption and the transport of K3R for guidance in determining its clinical dose.

Caco- 2 cells belong to the human colon adenocarcinoma cell line; they can spontaneously differentiate from small intestinal epithelial cells and have similar shapes and functions. Caco-2 monolayer cells are closely linked and express multiple membrane transporters, such as P-glycoprotein (P-gp) and multidrug resistance protein (MRP). Thus, the Caco-2 monolayer model is used by the US FDA to simulate human intestinal absorption and is widely used in drug research and development. ${ }^{15-18}$ In situ intestinal perfusion techniques in rats can maintain a supply of blood to intact intestines; it has been reported that oral drug absorption in rats and in the human body have a linear correlation. ${ }^{19}$ Therefore, the combined study of K3R absorption in the Caco-2 monolayer cell model and the rat in situ intestinal perfusion model could increase understanding of the absorption characteristics of K3R.

Crossing the BBB is a key step in the treatment of CNS disease by K3R. ${ }^{20}$ Thus, in this study, the immortalized mouse 
brain endothelial cell line bEnd. 3 was used to establish an in vitro BBB model. Because bEnd. 3 has the characteristics of low fluorescein sodium permeability and high expression of tight junction proteins, it is a commonly used BBB model. ${ }^{21,22}$ The study of K3R cross-BBB behavior in the bEnd.3 monolayer cell model is beneficial to further study the pharmacological activity of K3R.

Next, the pharmacological effects of K3R on neuronal cells were studied using pheochromocytoma (PC12) cells. PC12 cells have many similar characteristics to dopaminergic neurons, such as dopamine (DA) synthesis, metabolism and transport. Thus, they are widely used as an in vitro dopaminergic neuronal model $^{23}$ to study nerve injury and protection. Rotenone is highly lipophilic and binds to dopaminergic neurons to specifically inhibit the activity of mitochondrial complex I, thereby causing neuronal cell degeneration and death, which is characteristic of Parkinson's disease (PD). ${ }^{24}$

In this study, we investigated the transport of K3R in the Caco-2 absorption model, the rat orthotopic intestinal perfusion model, and the bEnd.3 BBB model, as well as its protection effects on the rotenone-induced PC12 cell model.

\section{Results}

\subsection{Quantitative analysis of K3R in transport solution}

The HPLC method to test K3R transportation was verified in this research. The standard curve showed a good linear relationship ( $r \geq 0.9998$ ), and the linear equation was $Y=102836 X$
- 3835.7 ( $X$ is the concentration and $Y$ is the peak area) in the range of 0.03 to $10 \mu \mathrm{g} \mathrm{mL} \mathrm{m}^{-1}$ for K3R (the limit of detection was $10 \mathrm{ng} \mathrm{mL} \mathrm{m}^{-1}$ and the limit of quantity was $38 \mathrm{ng} \mathrm{mL}^{-1}$ ).

The repeatability of the method was evaluated by testing K3R solutions at three concentration levels (high, middle and low); each concentration was tested in three batches. The RSD value of repeatability was less than $1.93 \%$, which showed the high repeatability of the method.

The precision of the method was evaluated by testing the K3R solutions at three different concentration levels (high, middle and low). The experiment was repeated five times on the same day to afford intra-day precision. The three concentrations of solution were prepared and tested five times on three consecutive days to determine the inter-day precision. The RSD of the intra-day precision was less than $0.38 \%$ and that of the inter-day precision was less than $1.86 \%$, which showed that the HPLC method to test K3R in the transport solution was highly precise.

The K3R solution was stored at $37^{\circ} \mathrm{C}$ and analyzed at $0,4,8$ and $12 \mathrm{~h}$, respectively. The relative standard deviation of the peak area was taken as a measure of stability and was less than $0.14 \%$. This result showed that the K3R solution has good stability under these conditions.

\subsection{Cytotoxicity assay}

The influence of K3R on the viability of Caco-2 cells, bEnd.3 cells and PC12 cells was investigated in this research. The data showed that there was no significant cytotoxicity on Caco-2
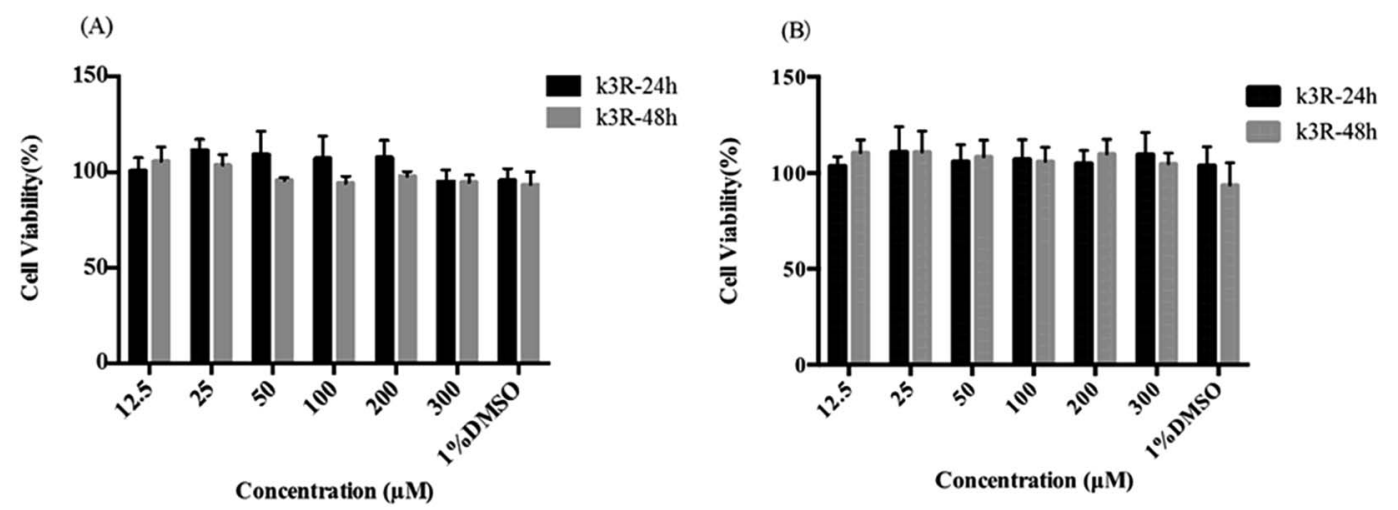

(C)

(D)
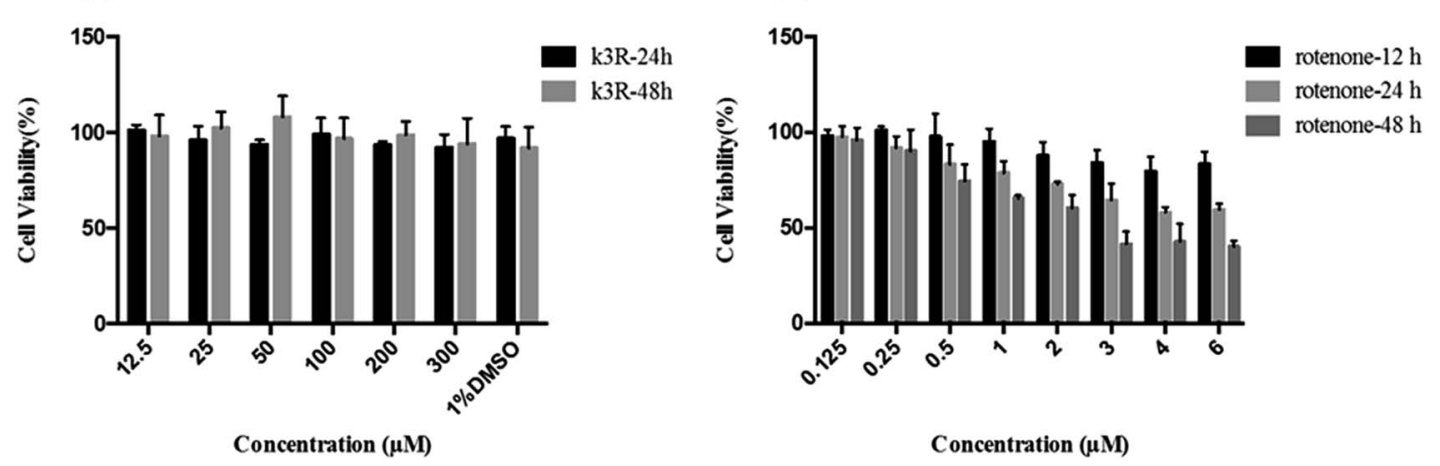

Fig. 1 Cell viability of (A) Caco-2 cells, (B) bEnd. 3 cells and (C) PC12 cells affected by K3R at $24 \mathrm{~h}$ and $48 \mathrm{~h}$. And (D) PC12 cells were damaged after the addition of rotenone. Values are presented as mean \pm SD $(n=4)$. 
cells, bEnd. 3 cells or PC12 cells (the survival rate was more than $90 \%$, Fig. 1) when the concentration of K3R was $12.5 \mu \mathrm{M}$ to 300 $\mu \mathrm{M}$ within $48 \mathrm{~h}$ of incubation. Also, 1\% dimethyl sulfoxide (DMSO) had no effect on cell viability. In addition, Féres CA et $a{ }^{25}$ carried out acute and chronic toxicological studies of rutin, a dry extract from Dimorphandra mollis, in rodents; this study suggested that the extract is safe in a $1000 \mathrm{mg} \mathrm{kg}^{-1}$ dose. Therefore, we expected that the concentration of K3R in the follow-up experiments would not affect the results due to toxicity on cells and mucosa.

The toxicity of rotenone to PC12 cells increased with increasing concentration and time within $48 \mathrm{~h}$. When the concentration of rotenone was greater than $1 \mu \mathrm{M}$, the survival rate of PC12 cells decreased significantly after $24 \mathrm{~h}$ treatment (Fig. 1D).

\subsection{Transport experiments of $K 3 R$ in the Caco-2 cell monolayer}

As shown in Fig. 2A, after being cultured for 21 days, Caco-2 cells can form a tightly connected monolayer with good integrity. Transmission electron microscopy (TEM) images (Fig. 2B) showed that the monolayer cells had characteristics of intestinal epithelial cells, such as the differentiation of microvilli on their tops and the formation of cell desmosomes between cells. This indicated that the monolayer of Caco- 2 cells could be used to simulate the intestinal absorption process of drugs in vivo. For apical (AP) to basolateral (BL) transport, the apparent permeability coefficients $\left(P_{\text {app }}\right)$ of K3R at different $\mathrm{pH}$ values are shown in Fig. 2C. There was no significant difference in $P_{\text {app }}$ at different $\mathrm{pH}$ values. $P_{\mathrm{app}(\mathrm{AP} \rightarrow \mathrm{BL})}$ increased with increasing concentration of K3R from $50 \mu \mathrm{M}$ to $200 \mu \mathrm{M}$ (Fig. 2D), indicating that the $P_{\text {app }}$ of K3R was concentration dependent. The $P_{\text {app }(\mathrm{AP} \rightarrow \mathrm{BL})}$ of $\mathrm{K} 3 \mathrm{R}$ was about 1.09 to $1.49 \times 10^{-6} \mathrm{~cm} \mathrm{~s}^{-1}$. All these results indicated that the absorption of K3R was consistent with the passive diffusion mechanism, ${ }^{\mathbf{1 6 , 2 6}}$ with a relatively moderate absorptive capacity. ${ }^{27}$

The result of bidirectional transport of K3R in the Caco-2 cell monolayer is shown in Fig. 2E. Although the $P_{\mathrm{app}(\mathrm{AP} \rightarrow \mathrm{BL})}$ of K3R was smaller than the $P_{\mathrm{app}(\mathrm{BL} \rightarrow \mathrm{AP})}$, the efflux rate (ER) was 1.25, which is less than 1.5. In order to determine whether an efflux effect occurred during the absorption process, the P-gp inhibitor verapamil was added to investigate the bidirectional
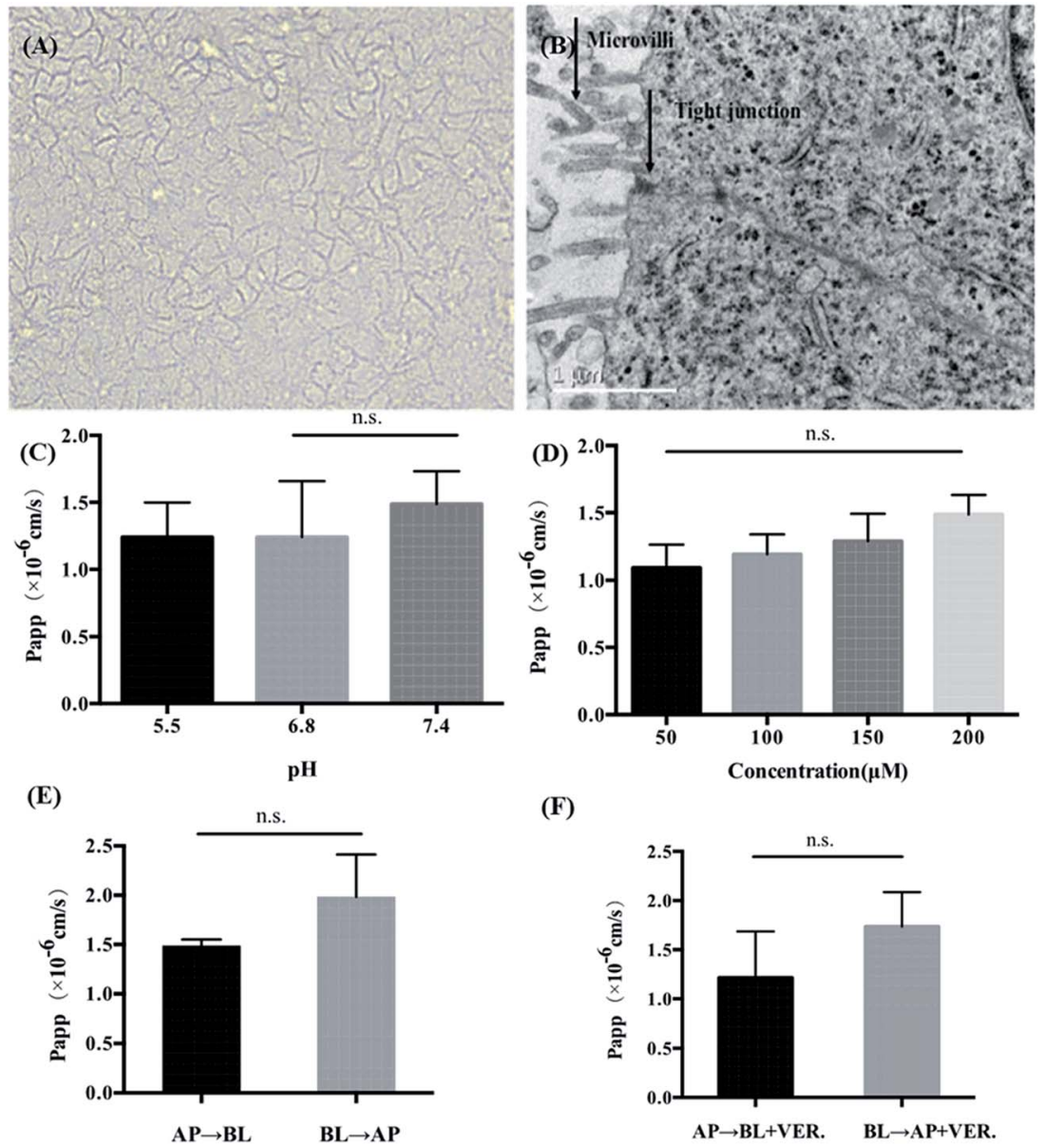

(F)

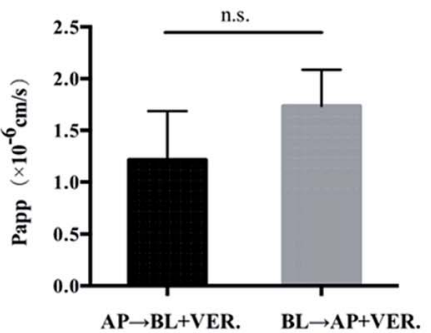

Fig. 2 Transport of K3R in Caco-2 cells. (A) Inverted microscope and (B) TEM image of Caco-2 monolayer cells. (C) Effects of pH on transport at a K3R concentration of $200 \mu \mathrm{M}$. (D) Effects of K3R concentration on transport. (E) $P_{\text {app }}$ of K3R bidirectional transport at a K3R concentration of $200 \mu \mathrm{M}$. (F) Effects of verapamil (VER.) on K3R bidirectional transport at a K3R concentration of $200 \mu \mathrm{M}$. Values are presented as mean \pm SD $(n=$ 3). Notes: ${ }^{*}, P<0.05 ; * *, P<0.01$; n.s., no significance. 
Table 1 Absorption parameters of K3R in different intestinal segments of rats (mean $\pm S D, n=6$ )

\begin{tabular}{lllll}
\hline Segment & $K_{\mathrm{a}}\left(\mathrm{h}^{-1}\right)$ & $t_{1 / 2}(\mathrm{~h})$ & $P(\%)$ & $P_{\text {app }}\left(\times 10^{-6}, \mathrm{~cm} \mathrm{~s}^{-1}\right)$ \\
\hline Duodenum & $0.22 \pm 0.09$ & $3.60 \pm 1.55$ & $27.50 \pm 6.40$ & $4.21 \pm 1.68$ \\
Jejunum & $0.24 \pm 0.12$ & $3.59 \pm 1.60$ & $23.67 \pm 10.23$ & $4.54 \pm 2.17$ \\
Ileum & $0.23 \pm 0.11$ & $3.66 \pm 1.62$ & $23.65 \pm 14.16$ & $4.39 \pm 2.17$ \\
Colon & $0.16 \pm 0.07$ & $5.16 \pm 2.57$ & $21.81 \pm 13.09$ & $4.05 \pm 1.84$
\end{tabular}

transport of K3R. The results (Fig. 2F) showed that after adding verapamil, the $P_{\mathrm{app}(\mathrm{BL} \rightarrow \mathrm{AP})}$ of $\mathrm{K} 3 \mathrm{R}$ was still higher than the $P_{\text {app(AP } \rightarrow \text { BL) }}$ and was close to the result for bidirectional transport without inhibitor. It can be concluded that $\mathrm{K} 3 \mathrm{R}$ is not a substrate for P-gp, and the transport process may involve the effects of other efflux proteins, such as MRP. ${ }^{28,29}$

\subsection{In situ rat intestinal perfusion experiment of K3R}

The absorption parameters of K3R during in situ intestinal perfusion experiments in rats are shown in Table 1; it was found that K3R was absorbed in all four rat intestine segments. The absorption rate constant $\left(K_{\mathrm{a}}\right)$, half-life $\left(t_{1 / 2}\right)$, first hour absorption rate $(P)$ and $P_{\text {app }}$ of the duodenum, jejunum and ileum were very close. Although the $K_{\mathrm{a}}, P$ and $P_{\text {app }}$ values of the colonic segment were slightly smaller than those of the first three intestines and the $t_{1 / 2}$ was slightly longer, there was no significant difference. These results indicated that K3R was absorbed in the intestine without specific absorption sites. The logarithm of the residual dose of $\mathrm{K} 3 \mathrm{R}$ in the intestine was plotted against the sampling time with a linear correlation and a coefficient $r>$ 0.9 , suggesting that $\mathrm{K} 3 \mathrm{R}$ absorption in the intestine follows first-order kinetics (Fig. 3).

\subsection{Transport of $\mathrm{K} 3 \mathrm{R}$ across the BBB in vitro}

bEend.3 cells were inoculated on a transwell plate, and the transepithelial electrical resistance (TEER) was measured after five days. The TEER values gradually increased and tended to be stable during the culture process. The cell formed a tight, integrated monolayer similar to the brain endothelial structure when the TEER reached $200 \Omega \mathrm{cm}^{-2}$. Then, the integrity of the cell monolayer was evaluated with fluorescein sodium-HBSS

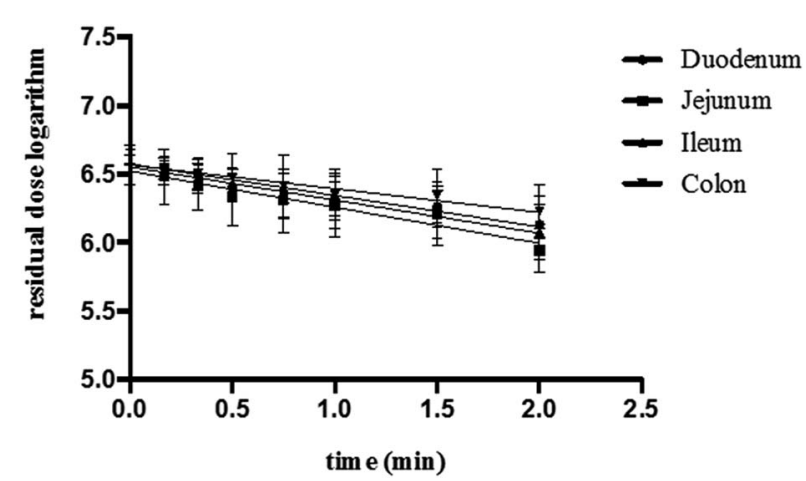

Fig. 3 The logarithm of the residual dose of K3R in different intestinal segments of rats (mean $\pm S D, n=6$ ). solution $\left(2 \mu \mathrm{g} \mathrm{mL}^{-1}\right)$. The result showed that the $P_{\text {app }}$ of fluorescein sodium in the direction from the inner chamber to the outer chamber was $(9.970 \pm 0.574) \times 10^{-6} \mathrm{~cm} \mathrm{~s}^{-1}$, indicating that the tightness of the $\mathrm{BBB}$ cell model was good and that it could be used in BBB-crossing experiments with K3R. ${ }^{30}$

K3R solutions with concentrations of 100,200 , or $300 \mu \mathrm{M}$ were respectively added to the inner chamber, and K3R could be detected in the outer chamber; this indicated that K3R could cross the BBB. The transport ratio of $\mathrm{K} 3 \mathrm{R}$ was proportional to the time and concentration (Fig. 4). $4 \mathrm{~h}$ later, the transport ratio could reach $45-65 \%$ without saturation. $P_{\text {app }}$ was also proportional to the concentration within $(1.34 \pm 0.26)$ to $(1.98 \pm 0.13)$ $\times 10^{-5} \mathrm{~cm} \mathrm{~s}^{-1}$. These results indicated that K3R could cross the $\mathrm{BBB}$ and enter brain tissue.

\subsection{Protective effects of K3R on rotenone-induced PC12 cell damage}

Compared with the control group (untreated), the cell survival rate of PC12 cells in the model group significantly decreased to (78.6 \pm 6.2)\% after treatment with $1 \mu \mathrm{M}$ rotenone for $24 \mathrm{~h}$ (Fig. 5A). After incubation with 100, 200 and $250 \mu \mathrm{M} \mathrm{K3R}$ for 4 hours, $1 \mu \mathrm{M}$ rotenone was added; the PC12 cell viability increased compared with the model group. When the K3R concentration was $250 \mu \mathrm{M}$, the survival rate of PC12 cells was $(87.16 \pm 2.8) \%$, indicating that $\mathrm{K} 3 \mathrm{R}$ was able to inhibit rotenone-induced injury in PC12 cells.

Lactate dehydrogenase $(\mathrm{LDH})$ is rapidly released into the culture medium when the cell membrane breaks down. The $\mathrm{LDH}$ release in the model group was $(142.7 \pm 2.5) \%$. The releases of LDH in PC12 cells after preincubation with K3R (100, $200,250 \mu \mathrm{M})$ were $(114.8 \pm 2.9) \%,(105.1 \pm 5.8) \%$ and $(103.1 \pm$ $2.1) \%$, respectively; this indicated that the release of $\mathrm{LDH}$ significantly decreased with increasing K3R concentration (Fig. 5B).

The higher the intracellular reactive oxygen species (ROS) level, the more severe the oxidative stress injury. In this study, PC12 cells were treated with rotenone; the level of ROS was 2 times that of the control group, indicating that the toxicity of rotenone to the cells was related to oxidative stress injury. Pretreatment with K3R $(100,200$, and $250 \mu \mathrm{M})$ significantly inhibited the production of ROS, which decreased to (131.7 \pm $11.3) \%,(80.3 \pm 3.8) \%$ and $(60.1 \pm 12.1) \%$, respectively. The results indicated that K3R had good antioxidant effects. ${ }^{31,32}$ The antioxidant activity of K3R contributes to the inhibition of ROS increase in rotenone-induced PC12 cells.

Compared with the control group, the mitochondrial membrane potential of PC12 cells was reduced by $20 \%$ after 
(A)



(B)

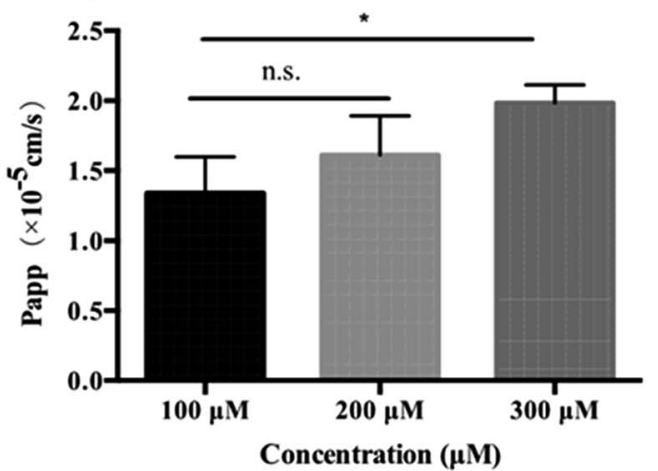

Fig. 4 K3R BBB crossing in vitro. (A) Transport ratio of K3R across the BBB; (B) transport $P_{\text {app }}$ of K3R across the BBB. Values are presented as mean $\pm \mathrm{SD}(n=3)$. Notes: $*, P<0.05 ; * *, P<0.01 ;$ n.S., no significance.
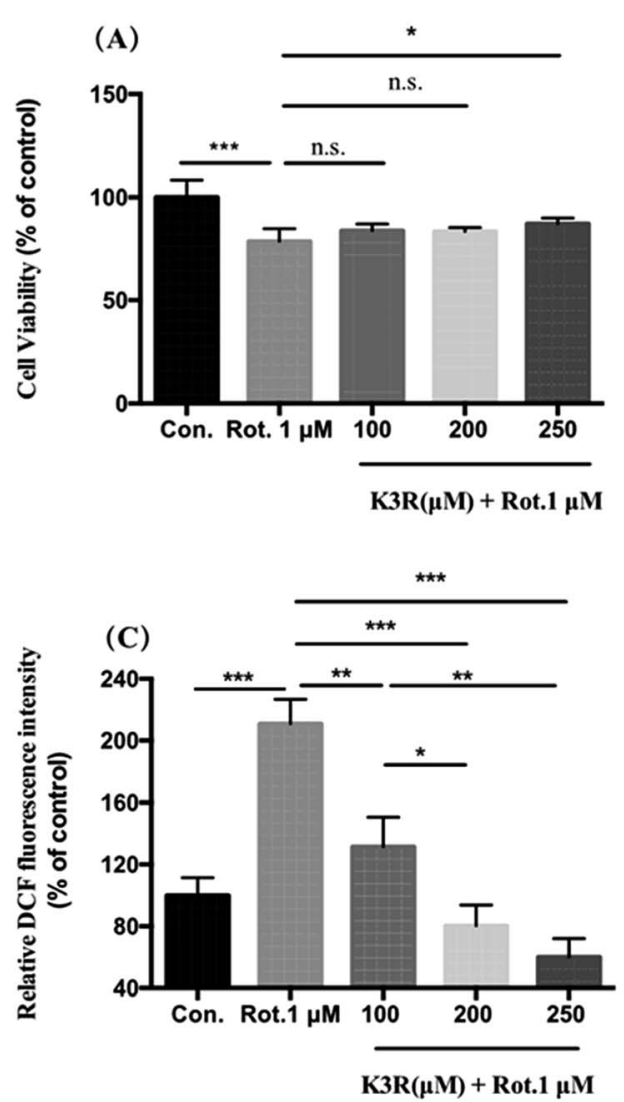
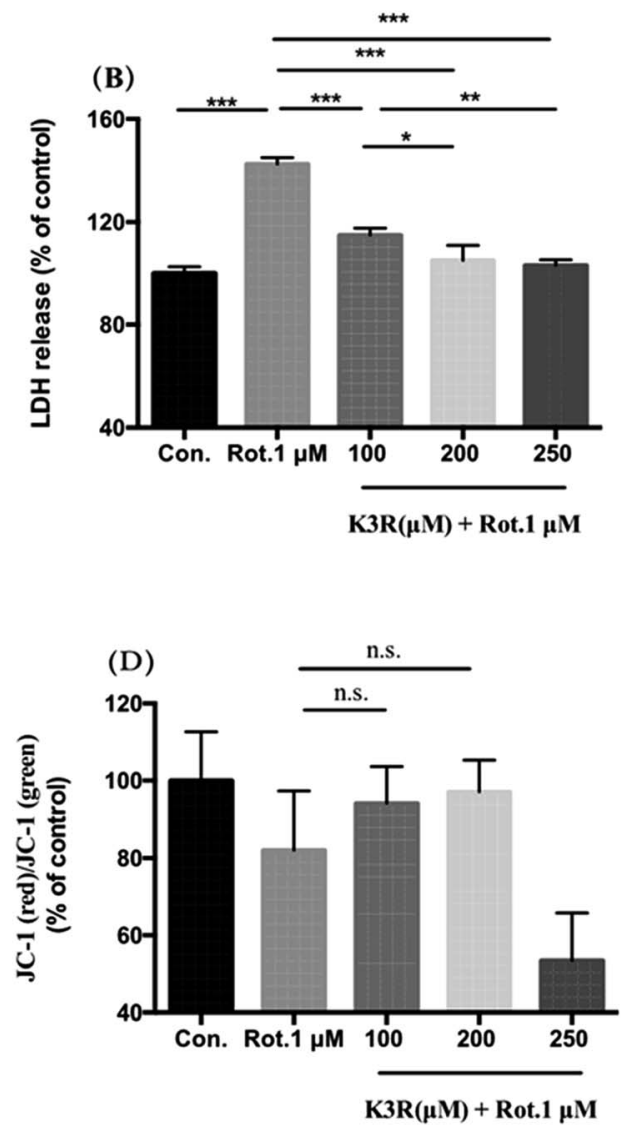

Fig. 5 Effects of K3R on rotenone-induced PC12 cell damage. The cells were treated with K3R at different concentrations for $4 \mathrm{~h}$ and then treated with $1 \mu \mathrm{M}$ rotenone for $24 \mathrm{~h}$. (A) Cell viability assayed by SRB; (B) released lactate dehydrogenase activity; (C) reactive oxygen species level and (D) mitochondrial membrane potential determination by flow cytometry. Notes: $*, P<0.05 ; * *, P<0.01$; n.s., no significance.

treatment with $1 \mu \mathrm{M}$ rotenone for 24 hours. Pretreatment for 4 hours with K3R at concentrations of 100 and $200 \mu \mathrm{M}$ increased the cell membrane potentials to $(94.2 \pm 4.9) \%$ and $(97.1 \pm 2.8) \%$, respectively. However, when the K3R concentration was $250 \mu \mathrm{M}$, the mitochondrial membrane potential of the cells was lower than that of the model group; the reason for this is not clear.

\section{Discussion}

It has been reported that the low water solubility and permeability of flavonoids limit their absorption and bioavailability in vivo. ${ }^{\mathbf{1 6}} \mathrm{K} 3 \mathrm{R}$ has prominent biological activity in the CNS; thus, it is necessary to study the absorption properties of $\mathrm{K} 3 \mathrm{R}$ and its ability to cross the BBB. The $P_{\mathrm{app}}$ value is important to predict 
the permeability of drugs. A drug is considered to be well absorbed (20-80\%) when the $P_{\text {app }}$ is greater than $1 \times 10^{-6} \mathrm{~cm}$ $\mathrm{s}^{-1}$ in Caco-2 cells. ${ }^{27}$ As a flavonoid glycoside compound, the $P_{\text {app(AP } \rightarrow \text { BL) }}$ of K3R of (1.09 to 1.49) $\times 10^{-6} \mathrm{~cm} \mathrm{~s}^{-1}$ in Caco-2 cells showed relatively moderate absorption (Fig. 2). The results were subsequently confirmed by an in vivo intestinal perfusion experiment; the $P_{\text {app }}$ was found to be $(4.05$ to 4.54$) \times 10^{-6} \mathrm{~cm}$ $\mathrm{s}^{-1}$. This is mainly because the glycosyl structure of K3R can promote absorption and increase bioavailability, as pointed out by Hollman et $a .^{33}$ and Walle et $a l .^{34}$ when they studied the absorption of quercetin glycosides and quercetin. However, the actual absorption of drugs should be based on their pharmacokinetics in vivo, and follow-up experiments will be performed. The fluxes of K3R were linearly associated with time, and no saturation phenomenon occurred; this indicates that the transport of K3R was mainly passive. ${ }^{35}$ The ER of K3R was less than 1.5, and the P-gp inhibitor verapamil did not increase the $P_{\text {app(AP } \rightarrow \text { BL) }}$ of K3R; this showed that the transport of K3R was not affected by the $\mathrm{ABC}$ efflux transporter P-gp. In order to identify the other specific protein involved in the process, further experiments must be conducted.

Youdim studied the behavior of citrus flavonoids across the $\mathrm{BBB}$. The results showed that citrus flavonoids traversed the BBB in the form of metabolites and showed a time-and dosedependent passive transcellular diffusion process. The amount of permeation is related to the lipophilicity of the metabolites, and the main metabolites to cross the BBB were glucuronidated flavonoids. ${ }^{36}$ In this research, the behavior of K3R across the BBB was studied, and the permeability and $P_{\text {app }}$ values showed concentration and time dependence, which is consistent with reported results. It has also been reported that glycosides formed by the hydrolysis of the $O$-glycosidic bonds of flavonoids have better lipophilicity and permeability than their parent glycosides, which is favorable for transmembrane transport. $^{37}$ Thus, K3R has a good ability to cross the BBB.

Studies have shown that the damage mechanism of rotenone to nerve cells involves the production of ROS and the loss of mitochondrial function. ${ }^{38}$ In our study, the toxicity of rotenone to cells increased with increasing concentration and time (Fig. 2D). By cytotoxicity and $\mathrm{LDH}$ release assays, it was confirmed that the survival rate of rotenone-induced PC12 cells significantly increased after pretreatment with K3R. The LDH leakage was reduced by $40 \%$, and the protective effect was concentration dependent (Fig. 5), consistent with our previous experimental results. ${ }^{39}$ It was further confirmed that the protective effects of K3R on PC12 cells were achieved by preventing the decrease of the mitochondrial membrane potential and inhibiting the production of intracellular ROS. This was due to the high scavenging free radical ability and antioxidant activity of K3R. ${ }^{31}$ It has been reported that the presence of K3Rspecific sugar side chains (rutose) enhances its neuroprotective activity. ${ }^{\mathbf{4 0 , 4 1}}$ It was reported that the biological activities of quercetin and its glycoside derivatives are related to their metabolites. ${ }^{42}$ Therefore, many biological activities of K3R are related to its metabolites in vivo. The metabolites may be directly involved in the biological process of $\mathrm{K} 3 \mathrm{R}$ and play a role in its efficacy.

\section{Materials and methods}

\subsection{Materials}

K3R (purity 98\%) was obtained from the ZeLang group (China). Sulforhodamine B (SRB), ethylene diamine tetraacetic acid (EDTA) and verapamil hydrochloride were obtained from Sigma Chemical Co. (USA). The high performance liquid chromatography (HPLC) solvents methanol, acetonitrile and formic acid were obtained from Fisher (USA). All other chemicals were of analytical grade or HPLC grade.

Caco- 2 cells and bEnd. 3 cells were obtained from American Type Culture Collection (ATCC, USA). PC12 cells were purchased from the cell bank of the Chinese Academy of Sciences. Minimum essential medium (MEM), Dulbecco's Modified Eagle's Medium (DMEM), RPMI 1640, sodium pyruvate solution, penicillin-streptomycin, non-essential amino acid and Hank's balanced salt solution (HBSS) were purchased from M\&C Gene Co., Ltd. (China). Fetal bovine serum (FBS) was obtained from Gibco Co. (USA). Transwell culture plates and dishes were obtained from Corning Inc. (USA).

Male Sprague-Dawley (SD) rats, 8 weeks old, $250 \pm 20 \mathrm{~g}$, were obtained from Peking University Health Science Center. Animal certificate number: SCXK (Jing) 2016-0010. The rats were housed in standard conditions at room temperature $\left(22 \pm 2{ }^{\circ} \mathrm{C}\right)$ and humidity ( $55 \pm 5 \%$ ). The rats were acclimated for at least 3 days and had free access to food and water.

All the animal experiments were performed in compliance with the relevant laws and institutional guidelines of the Institutional Animal Care and Use Committee of Peking University Health Science Center and were approved by the Institutional Animal Care and Use Committee of Peking University Health Science Center (Beijing, China).

\subsection{Quantitative analysis of K3R}

4.2.1 HPLC conditions. The concentration of K3R was determined using a Shimadzu (Japan) HPLC system equipped with an LC-20AT pump, SPD-20A UV detector, SIL-20A autosampler and Alltima HP-C18 column $(250 \mathrm{~mm} \times 4.6 \mathrm{~mm}, 5 \mu \mathrm{m}$, Alltech, USA). The mobile phase was acetonitrile : water $(0.3 \%$ formic acid added) in a ratio of $20: 80(\mathrm{v} / \mathrm{v})$. The UV detection wavelength was $345 \mathrm{~nm}$, the flow rate was $1.0 \mathrm{~mL} \mathrm{~min}{ }^{-1}$, and the injection volume was $20 \mu \mathrm{L}$.

4.2.2 Analytical method validation. Experiments, including linearity, precision (intra-day precision, day precision and repeatability) and accuracy of $\mathrm{K} 3 \mathrm{R}$, were carried out to validate the quality inspection of the methods. ${ }^{43}$

\subsection{Cell culture}

Caco-2 cells were grown in MEM medium with 10\% FBS, 1\% penicillin-streptomycin, $1 \%$ pyruvic acid sodium and $1 \%$ nonessential amino acid. bEnd.3 cells were cultured in DMEM medium containing $10 \%$ FBS and $1 \%$ penicillin-streptomycin. PC12 cells were grown in RPMI 1640 medium containing 10\% FBS and $1 \%$ penicillin-streptomycin. The cells mentioned above were placed in a $37{ }^{\circ} \mathrm{C}$ humidified incubator with $5 \% \mathrm{CO}_{2}$ atmosphere. When the cells in the culture flask were $70-80 \%$ 
fused, they were passaged at a ratio of $1: 3$ and digested with $0.25 \%$ trypsin (containing $0.02 \%$ EDTA).

\subsection{Cytotoxicity assay}

The influence of K3R on the viability of Caco-2 cells, bEnd.3 cells and PC12 cells was detected by the sulforhodamine B (SRB) method. At the same time, the damage of PC12 cells induced by rotenone was investigated. K3R or rotenone was first dissolved in DMSO and diluted with PBS to the required concentration before use. The concentration of DMSO in the cell culture medium was controlled to $1 \%$ or below. Caco- 2 cells, bEnd. 3 cells and PC12 cells were seeded in 96-well plates at a density of $1.5 \times 10^{4}$ cells per $\mathrm{mL}$ and then incubated in an incubator for $24 \mathrm{~h}$, respectively. After that, the cells were treated with K3R for 24 or $48 \mathrm{~h}$, and another group was treated with rotenone for 12, 24 and $48 \mathrm{~h}$ at different concentrations. The culture medium was removed and monitored by the SRB method; then, the plate was placed on an iMark microplate reader (Bio-Rad Laboratories, CA, USA) at a wavelength of $540 \mathrm{~nm}$ to determine the optical density (OD) values. ${ }^{\mathbf{4 4}}$ The cell viability was calculated using the following formula: cell viability $(\%)=\left(\mathrm{OD}_{\text {treated }}\right)$ $\left.\mathrm{OD}_{\text {control }}\right) \times 100 \%$.

\subsection{Transport experiments on the Caco-2 monolayer cell model of K3R}

4.5.1 Validation of the Caco-2 monolayer cell model. For the transport experiments, Caco- 2 cells were placed in $4 \times 10^{5}$ cells per $\mathrm{mL}$ cell suspensions, and $0.5 \mathrm{~mL}$ was inoculated into 12-well transwell culture chambers. $1.5 \mathrm{~mL}$ fresh culture medium was added to the outer chamber, and the cells were placed into a $37{ }^{\circ} \mathrm{C}, 5 \% \mathrm{CO}_{2}$ incubator. The culture medium in each well was replaced once every two days and once every day two weeks after the inoculation of the cells. At the same time, the transepithelial electrical resistance (TEER) was measured every two days using a Millicell-ERS electrode (Millipore, Billerica, USA). After incubation for 21 days, the wells with TEER values of more than $500 \Omega \mathrm{cm}^{-2}$ were selected for the transport experiments. ${ }^{\mathbf{1 6 , 4 5}}$

The Caco-2 cell monolayers were cultured continuously for 21 days, and the morphology of the monolayer membrane was observed with an inverted microscope (AMG EVOS, USA). The monolayer cells were scraped gently and maintained overnight at $4{ }^{\circ} \mathrm{C}$. The monolayer cells were finally dehydrated with different concentrations of ethanol solution and observed by TEM (JEOL Ltd., Japan).

4.5.2 Transport experiments on the Caco-2 monolayer cell model of K3R. Before the transport experiments, the culture medium was removed from the mature Caco- 2 cell monolayers, and the monolayers were washed twice with HBSS solution $(\mathrm{pH}$ $7.4,37{ }^{\circ} \mathrm{C}$ ). For apical to basolateral transport, $0.5 \mathrm{~mL} \mathrm{K3R}$ solution $(200 \mu \mathrm{M})$ was added to the apical membrane, and 1.5 $\mathrm{mL}$ HBSS blank solution was added to the basolateral membrane. For BL to apical AP transport, $1.5 \mathrm{~mL}$ drug solution was added to the basolateral membrane, and $0.5 \mathrm{~mL}$ HBSS blank solution was added to the apical membrane. The culture plates were placed in an air bath in the dark $\left(37^{\circ} \mathrm{C}, 50 \mathrm{rpm}\right)$, and
$0.2 \mathrm{~mL}$ of solution was sampled from the AP (BL $\rightarrow$ AP direction) or the BL (AP $\rightarrow$ BL direction) at 30, 60, 90, 120, 150, 180 and $240 \mathrm{~min}$, respectively. The same volume of blank HBSS solution was added after each sample was removed. The removed samples were stored at $-20{ }^{\circ} \mathrm{C}$ until analysis.

Similarly, the effects of drug concentration, $\mathrm{pH}$ (5.5, 6.8 and 7.4) and P-gp inhibitors $\left(20 \mu \mathrm{g} \mathrm{mL} \mathrm{mL}^{-1}\right.$ verapamil) on the transport of K3R were evaluated, respectively. The $P_{\text {app }}$ values under different conditions were calculated.

4.5.3 Data analysis. The apparent permeability coefficient $\left(P_{\text {app }}, \mathrm{cm} \mathrm{s}^{-1}\right)$ and efflux rate (ER) were calculated using eqn (1) and (2).

$$
P_{\text {app }}=\frac{\Delta Q}{\Delta t A C_{0}}
$$

where $\mathrm{d} Q / \mathrm{d} t$ is the transport rate $\left(\mu \mathrm{g} \mathrm{s}^{-1}\right), A$ is the area of the polycarbonate membrane per well $\left(1.12 \mathrm{~cm}^{2}\right)$, and $C_{0}$ is the initial concentration of the drug $\left(\mu \mathrm{g} \mathrm{mL}{ }^{-1}\right)$.

$$
\mathrm{ER}=\frac{P_{\mathrm{app}(\mathrm{BL} \rightarrow \mathrm{AP})}}{P_{\mathrm{app}(\mathrm{AP} \rightarrow \mathrm{BL})}}
$$

where $P_{\text {app }(\mathrm{BL} \rightarrow \mathrm{AP})}$ is the $P_{\text {app }}$ from the basolateral cells to the apical cells and $P_{\text {app }(\mathrm{AP} \rightarrow \mathrm{BL})}$ is the $P_{\text {app }}$ from the apical cells to the basolateral cells.

\subsection{In situ rat intestinal perfusion experiment of K3R}

$\mathrm{SD}$ rats, after fasting for $15 \mathrm{~h}$ (free drinking water), were anesthetized and fixed by intraperitoneal injection of $20 \%$ urethane solution $\left(10 \mathrm{~mL} \mathrm{~kg}^{-1}\right)$. The abdominal cavity was opened along the abdominal line (about $3 \mathrm{~cm}$ ), and the required intestinal segment was located (about $10 \mathrm{~cm}$ ). A tube was inserted into the cuts at both ends of the intestine, ligated with surgical line, and then connected with a peristaltic pump (BT00-300M, LongerPump, China) to a recirculating reflux system. ${ }^{18}$ The contents of the intestine were rinsed with Krebs-Ringer buffer (KRB, 37 ${ }^{\circ} \mathrm{C}$ ) and the remaining KRB was expelled from the intestine with air. The K3R solution $\left(200 \mu \mathrm{M}\right.$; phenol red $\left.20 \mu \mathrm{g} \mathrm{mL} \mathrm{m}^{-1}\right)$ was

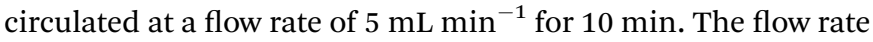
was adjusted to $2 \mathrm{~mL} \mathrm{~min}^{-1}$, and the intestinal perfusion was started. At 0, 10, 20, 30, 45, 60, 90 and $120 \mathrm{~min}, 0.5 \mathrm{~mL}$ perfusate was removed and $0.5 \mathrm{~mL}$ phenol red-containing $\mathrm{KRB}$ was added.

The KRB was prepared as follows. $\mathrm{NaCl}$ (7.80 g), $\mathrm{KCl}$ (0.35 g), $\mathrm{NaHCO}_{3}(1.37 \mathrm{~g}), \mathrm{NaH}_{2} \mathrm{PO}_{4}(0.32 \mathrm{~g}), \mathrm{MgCl}_{2}$ (0.02 g), and glucose $(1.4 \mathrm{~g})$ were dissolved in $900 \mathrm{~mL}$ water to afford solution I, and $\mathrm{CaCl}_{2}(0.37 \mathrm{~g})$ was dissolved in $100 \mathrm{~mL}$ water to afford solution II. Solution II was gently poured into solution I with stirring, and no precipitate was obtained. In addition, $20 \mu \mathrm{g} \mathrm{mL} \mathrm{m}^{-1}$ of phenol red was added to the KRB to correct the volume of the perfusate.

\subsection{Transport of $\mathrm{K} 3 \mathrm{R}$ across the $\mathrm{BBB}$ in vitro}

4.7.1 Quantitative analysis of $K 3 R$ in transport solution. For the BBB transport experiments, bEnd. 3 cells were placed in $2 \times 10^{5}$ cells per $\mathrm{mL}$ cell suspensions, and $0.5 \mathrm{~mL}$ was inoculated into 12-well transwell culture chambers. $1.5 \mathrm{~mL}$ fresh 
culture medium was added to the outer chamber, and the cells were then placed into a $37{ }^{\circ} \mathrm{C}, 5 \% \mathrm{CO}_{2}$ incubator. The culture medium in each well was replaced once every two days after the inoculation of the cells.

The BBB model was evaluated by fluorescein sodium permeability and TEER assays. The culture medium was removed from the bEnd. 3 cell monolayers and washed twice with HBSS solution ( $\left.\mathrm{pH} 7.4,37^{\circ} \mathrm{C}\right)$. For inner chamber to outer chamber transport, $0.5 \mathrm{~mL}$ fluorescein sodium solution $(2 \mu \mathrm{g}$ $\mathrm{mL}^{-1}$ ) was added to the inner chamber, and $1.5 \mathrm{~mL}$ HBSS blank solution was added to the outer chamber. $0.2 \mathrm{~mL}$ samples were removed from the outer chamber at 60, 90, 120,150,180, and $240 \mathrm{~min}$, respectively, and the same volume of HBSS solution was added. The fluorescence intensities of the fluorescein sodium samples were tested at $E_{\mathrm{x}} / E_{\mathrm{m}}=460 \mathrm{~nm} / 520 \mathrm{~nm}$ using a fluorescence spectrophotometer, and the $P_{\text {app }}$ was calculated according to the fluorescein sodium standard curve. The TEER of the monolayer cells was measured daily from the fifth day of bEnd. 3 cell inoculation; TEER $>200 \Omega \mathrm{cm}^{-2}$ (ref. 22) was used for the determination of fluorescein sodium across the BBB.

4.7.2 Transport of K3R across the BBB. The culture medium was removed from the bEnd. 3 cell monolayers and washed twice with HBSS solution ( $\left.\mathrm{pH} 7.4,37{ }^{\circ} \mathrm{C}\right)$. For inner chamber to outer chamber transport, $0.5 \mathrm{~mL}$ K3R solution (100, 200 , or $300 \mu \mathrm{M}$, respectively) was added to the inner chamber, and $1.5 \mathrm{~mL}$ HBSS blank solution was added to the outer chamber. The culture plates were placed in an air bath in the dark $\left(37^{\circ} \mathrm{C}, 50 \mathrm{rpm}\right)$, and $0.2 \mathrm{~mL}$ of solution was sampled from the BL at 10, 30, 60, 90, 120, 150, 180 and $240 \mathrm{~min}$, respectively. The same volume of blank HBSS solution was added after each sample was removed.

\subsection{Protective effects of K3R on rotenone-induced PC12 cell damage}

The PC12 cell injury model was established by treatment with 1 $\mu \mathrm{M}$ rotenone for $24 \mathrm{~h}$. The protective effects of K3R on rotenoneinduced PC12 cell injury were determined by analyzing the cell survival, LDH release, intracellular ROS and mitochondrial membrane potential data after preincubation of PC12 cells with different concentrations of K3R for $4 \mathrm{~h}$ followed by incubation with $1 \mu \mathrm{M}$ of rotenone for 24 hours. Cells treated with serumfree RPMI 1640 culture medium served as a control.

The cell survival was analyzed by the SRB method. Cell damage was evaluated by the release of $\mathrm{LDH}$ into the culture medium $^{46}$ according to the LDH kit instructions. The ROS assay was performed with a flow cytometer by adding DCFH-DA (10 $\mu \mathrm{M}$ ) and incubating at $37{ }^{\circ} \mathrm{C}$ for $20 \mathrm{~min}$ in the dark. ${ }^{47}$ The ratio of mitochondrial depolarization was measured by the ratio of red and green fluorescence caused by the binding of lipophilic fluorochrome JC-1 to the cells after the cell suspension was incubated with $\mathrm{JC}-1$ at $37{ }^{\circ} \mathrm{C}$ for $20 \mathrm{~min}$ in the dark. ${ }^{47,48}$

\subsection{Statistical analysis}

All values are expressed as mean \pm SD. Statistical differences ( $p$ $<0.05)$ were tested using one-way ANOVA with Bonferroni's multiple comparison test.

\section{Conclusions}

Kaempferol and its glycoside derivatives have a wide range of physiological and pharmacological activities. In vitro Caco-2 monolayer experiments and in situ intestinal perfusion studies indicate that the intestinal absorption of K3R occurs by passive diffusion with first-order kinetics without specific absorption sites in the intestine. The BBB transport of K3R was evaluated by a bEnd. 3 monolayer model, and the results showed that K3R has good transmembrane capacity. K3R protects nerve cells by preventing the decrease of mitochondrial membrane potential and inhibiting the production of ROS, thereby increasing the survival rate of cells with rotenone-induced PC12 injury and reducing the LDH permeability. In conclusion, the abovementioned findings suggest that K3R can be used to treat CNS disease in vivo due to its relatively good gastrointestinal absorption and BBB-crossing ability; the results also provide a scientific basis for the entry of K3R into brain tissue.

\section{Conflict of interest}

The authors declare no competing financial interest.

\section{Acknowledgements}

This work was supported by Science and Technology Major Projects: Significant New-Drugs Creation (No. 2012ZX09103201042).

\section{References}

1 A. A. Chinchansure, A. M. Korwar, M. J. Kulkarni and S. P. Joshi, RSC Adv., 2015, 5, 31113-31138.

2 A. Smeriglio, G. Mandalari, C. Bisignano, A. Filocamo, D. Barreca, E. Bellocco and D. Trombetta, Ind. Crops Prod., 2016, 83, 283-293.

3 Y. Wang, C. Tang and H. Zhang, J. Food Drug Anal., 2014, 23, 310-317.

4 A. Obmann, D. T. Tsendayush, M. Zehl, T. P. Vo, S. Purevsuren, D. Natsagdorj, S. Narantuya, C. Kletter and S. Glasl, J. Ethnopharmacol., 2010, 131, 555-561.

5 C. Dias, R. Domínguez-Perles, A. Aires, A. Teixeira, E. Rosa, A. Barros and M. J. Saavedra, LWT-Food Sci. Technol., 2015, 61, 25-32.

6 C. Grosso, J. Vinholes, L. R. Silva, P. G. D. Pinho, F. G. Rui, P. Valentão, A. K. Jäger and P. B. Andrade, Rev. Bras. Farmacogn., 2011, 21, 635-643.

7 N. Petpiroon, C. Suktap, S. Pongsamart, P. Chanvorachote and S. Sukrong, J. Nat. Med., 2015, 69, 340-348.

8 H. Zhang, N. Li, K. Li and P. Li, Mol. Med. Rep., 2014, 10, 3157.

9 R. Li, M. Guo, G. Zhang, X. Xu and Q. Li, J. Ethnopharmacol., 2006, 107, 143-150.

10 L. Yu, C. Chen, L. F. Wang, X. Kuang, K. Liu, H. Zhang and J. R. Du, PLoS One, 2013, 8, e55839.

11 A. Mira, S. Yamashita, Y. Katakura and K. Shimizu, Molecules, 2015, 20, 4813. 
12 J. L. Huang, S. T. Fu, Y. Y. Jiang, Y. B. Cao, M. L. Guo, Y. Wang and Z. Xu, Pharmacol., Biochem. Behav., 2007, 86, 741-748.

13 R. Ren, C. Shi, J. Cao, Y. Sun, X. Zhao, Y. Guo, C. Wang, H. Lei, H. Jiang and N. Ablat, Sci. Rep., 2016, 6, 22135.

14 N. Ablat, D. Lv, R. Ren, Y. Xiaokaiti, X. Ma, X. Zhao, Y. Sun, H. Lei, J. Xu and Y. Ma, Molecules, 2016, 21, 1107.

15 Y. Chen, Y. H. Zhao, X. B. Jia and M. Hu, Pharm. Res., 2008, 25, 2190-2199.

16 M. L. Zhu, X. L. Liang, L. J. Zhao, Z. G. Liao, G. W. Zhao, Y. C. Cao, J. Zhang and Y. Luo, J. Ethnopharmacol., 2013, 150, 553-559.

17 P. Artursson, K. Palm and K. Luthman, Adv. Drug Delivery Rev., 1996, 22, 67-84.

18 Q. Lin, L. Q. Ling, L. Guo, T. Gong, X. Sun and Z. R. Zhang, Acta Pharmacol. Sin., 2015, 36, 863-873.

19 W. L. Chiou and A. Barve, Pharm. Res., 1998, 15, 1792-1795.

20 W. M. Pardridge, Blood-Brain Barrier Drug Targeting: The Future of Brain Drug Development, Cambridge University Press, 2001.

21 T. Watanabe, S. Dohgu, F. Takata, T. Nishioku, A. Nakashima, K. Futagami, A. Yamauchi and Y. Kataoka, Biol. Pharm. Bull., 2013, 36, 492-495.

22 R. C. Brown, A. P. Morris and R. G. O'Neil, Brain Res., 2007, 1130, 17.

23 W. B. Liu, J. Zhou, Y. Qu, X. Li, C. T. Lu, K. L. Xie, X. L. Sun and Z. Fei, Neurochem. Int., 2010, 57, 206-215.

24 J. Huang, H. Liu, W. Gu, Z. Yan, Z. Xu, Y. Yang, X. Zhu and Y. Li, Biomaterials, 2006, 27, 937-946.

25 C. A. O. Féres, R. C. Madalosso, O. A. Rocha, J. P. V. Leite, T. M. D. P. Guimarães, V. P. P. Toledo and C. A. Tagliati, J. Ethnopharmacol., 2006, 108, 450-456.

26 R. Ding, Y. Jian, H. Kan, Z. Y. Liu, S. Liu, Z. Q. Liu and J. Zhou, Chin. Chem. Lett., 2016, 27, 915-919.

27 S. Yee, Pharm. Res., 1997, 14, 763-766.

28 X. Tian, X. Yang, K. Wang and X. Yang, Pharm. Res., 2006, 23, 1721-1728.

29 Y. Xie, J. Duan, Q. Fu, M. Xia, L. Zhang, G. Li, T. Wu and G. Ji, Eur. J. Pharm. Sci., 2015, 73, 1.

30 D. H. Xu, M. Yan, H. D. Li, P. F. Fang and Y. W. Liu, Eur. J. Pharmacol., 2012, 690, 68.
31 R. Domínguez-Perles, A. Guedes, M. Queiroz, A. M. Silva and A. I. R. N. Barros, Food Res. Int., 2016, 87, 92-102.

32 A. Fiorentino, B. D'Abrosca, S. Pacifico, A. Golino, C. Mastellone, P. Oriano and P. Monaco, Molecules, 2007, 12, 263-270.

33 P. C. Hollman, J. H. de Vries, S. D. van Leeuwen, M. J. Mengelers and M. B. Katan, Am. J. Clin. Nutr., 1995, 62, 1276.

34 T. Walle, Y. Otake, U. K. Walle and F. A. Wilson, J. Nutr., 2000, 130, 2658.

35 X. J. Tian, X. W. Yang, X. Yang and K. Wang, Int. J. Pharm., 2009, 367, 58-64.

36 K. A. Youdim, M. S. Dobbie, G. Kuhnle, A. R. Proteggente, N. J. Abbott and C. Rice-Evans, J. Neurochem., 2003, 85, 180.

37 A. Scheepens, K. Tan and J. W. Paxton, Genes Nutr., 2010, 5, 75-87.

38 M. F. Molinajimenez, M. I. Sanchezreus and J. Benedi, Eur. J. Pharmacol., 2003, 472, 81-87.

39 W. Qu, L. Fan, K. Yunchul, S. Ishikawa, S. M. M. Iguchiariga, X. P. Pu and H. Ariga, J. Pharmacol. Sci., 2009, 110, 191-200.

40 M. Nakayama, M. Aihara, Y. N. Chen, M. Araie, K. TomitaYokotani and T. Iwashina, Mol. Vision, 2011, 17, 1784.

$41 \mathrm{~S} . \mathrm{Wu}, \mathrm{W} . \mathrm{Xu}, \mathrm{F} . \mathrm{R}$. Wang and X. W. Yang, Molecules, 2015, 20, 18704-18716.

42 F. Lodi, R. Jimenez, L. Moreno, P. A. Kroon, P. W. Needs, D. A. Hughes, C. Santosbuelga, A. Gonzalezparamas, A. Cogolludo and R. Lopezsepulveda, Atherosclerosis, 2009, 204, 34-39.

43 H. Guo, A. H. Liu, L. Li and D. A. Guo, J. Pharm. Biomed. Anal., 2007, 43, 1000-1006.

44 Z. Z. Yang, J. Q. Li, Z. Z. Wang, D. W. Dong and X. R. Qi, Biomaterials, 2014, 35, 5226.

45 X. Chen, Y. Zhang, L. Yuan, H. Zhang, W. Dai, B. He, X. Wang and Q. Zhang, RSC Adv., 2015, 5, 104228-104238.

46 B. Y. Cao, Y. P. Yang, W. F. Luo, C. J. Mao, H. Rong, S. Xue, C. Jing and C. F. Liu, J. Ethnopharmacol., 2010, 131, 122-129.

47 D. Liang, A. T. Wang, Z. Z. Yang, Y. J. Liu and X. R. Qi, Mol. Pharmaceutics, 2015, 12, 2189.

48 A. T. Wang, D. S. Liang, Y. J. Liu and X. R. Qi, Biomaterials, 2015, 53, 160. 